\title{
Regulating expression of mistranslating tRNAs by readthrough RNA polymerase II transcription
}

Matthew D. Berg ${ }^{1,4, *}$, Joshua R. Isaacson², Ecaterina Cozma1 ${ }^{1}$, Julie Genereaux, Patrick Lajoie ${ }^{3}$, Judit Villén ${ }^{4}$ and Christopher J. Brandl ${ }^{1, *}$

${ }^{1}$ Department of Biochemistry, ${ }^{2}$ Department of Biology and ${ }^{3}$ Department of Anatomy and Cell Biology, The University of Western Ontario, London N6A 5C1, Canada

${ }^{4}$ Department of Genome Sciences, University of Washington, Seattle, WA 98195, USA

*Corresponding authors: Matthew Berg and Christopher Brandl, Department of Biochemistry, The University of Western Ontario, London, Ontario N6A 5C1, Canada, Email: mberg2@uwo.ca; cbrandl@uwo.ca

\section{Supplemental Material}

Supplemental Tables

Table S1. Oligonucleotides used in this study.

\begin{tabular}{|l|l|l|}
\hline Name & Sequence & Description \\
\hline $4588-1$ & CCCGGATCCGACGTTAAAGTATAGAGGT & GAL1pr \\
\hline XH5103 & CCCAAGCTTTAATACGCTTAACTGCTCATTG & GAL1pr \\
\hline XI6247 & TTTGGTACCCCTTTAGCTTCTCGACGTGG & HIS3pr \\
\hline XI6248 & TTTGCGGCCGCTCTTTGCCTTCGTTTATCTTGCC & HIS3pr \\
\hline UG5953 & TCTAAGCTTCGGACGATTGCCAACCGCCGAA & SUP17 \\
\hline UG5954 & CTGCAGAATTCCGCGGAAATTAGCACGGCC & SUP17 \\
\hline YA9566 & AAAGCGGCCGCGAACCGTGGCTAATAGG & SUP17 \\
\hline YA9567 & TTTGCGGCCGCTTTCCCCGAGAAAGCAACC & SUP17 \\
\hline YG4866 & TTTGGATCCGGGATCAACTCGAGTAATTCG & TetOpr \\
\hline YG4867 & GGGGCGGCCGCGTAATTTAGTGTGTGTATTTGTG & TetOpr \\
\hline YG4868 & CCCAAGCTTTCATTAGTAATTTAGTGTGTGTATTTGTG & TetOpr \\
\hline
\end{tabular}




\section{$\underline{\text { Supplemental Figures }}$}

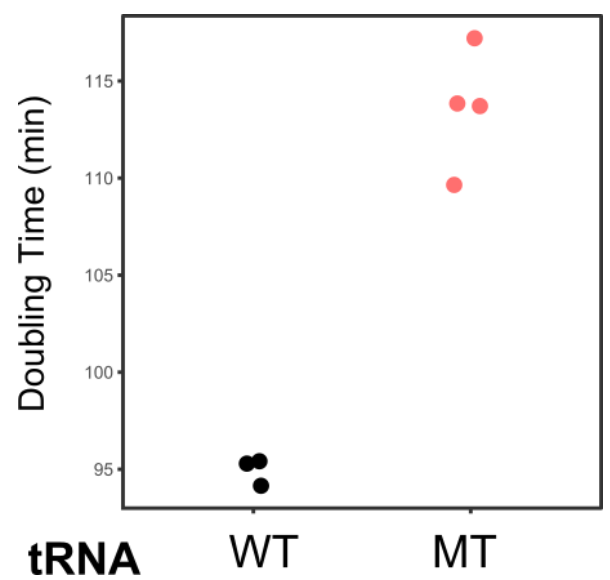

Figure S1. Expressing the GAL4 DNA binding domain alone does not inhibit the toxicity due to

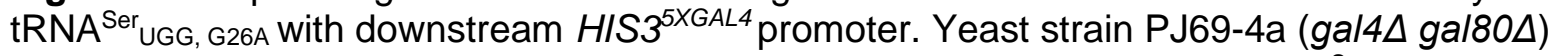
expressing the GAL4 DNA binding domain (pKW21) and either wild type tRNA ${ }^{\text {Ser }}$ regulated by a downstream HIS3 ${ }^{5 X G A L 4}$ promoter (WT) or mistranslating tRNA ${ }^{\text {Ser }}$ GGG, G26A (MT) regulated by a downstream HIS3 ${ }^{5 X G A L 4}$ promoter, were grown to stationary phase in minimal medium. Strains were diluted to an $\mathrm{OD}_{600}$ of 0.1 and grown for 24 hours at $30^{\circ} \mathrm{C}$ with agitation. $\mathrm{OD}_{600}$ was measured every 15 minutes and doubling time in minutes was calculated from the growth curves.

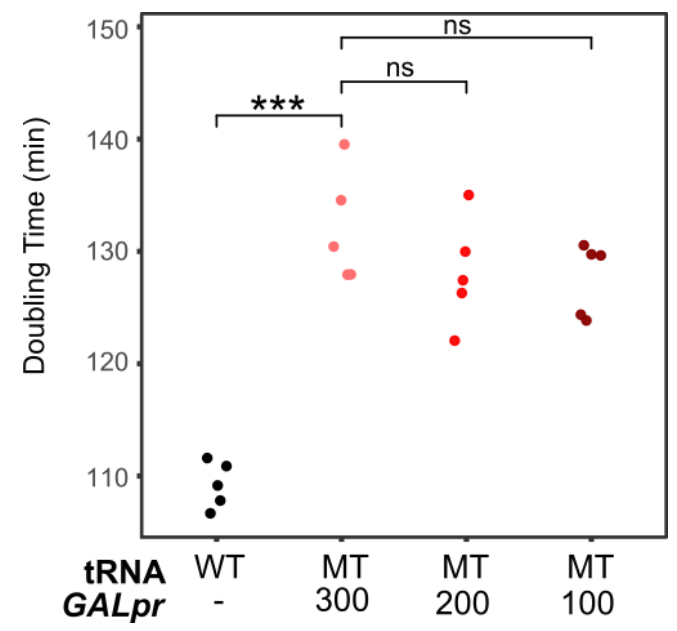

Figure S2. Distance between tRNA 3' end and GAL1 promoter does not affect toxicity in

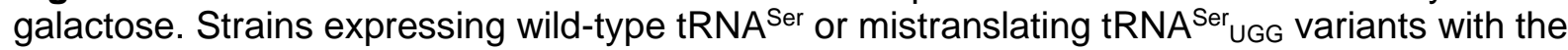
synthetic GAL1 promoter placed $300 \mathrm{bp}, 200 \mathrm{bp}$ or $100 \mathrm{bp}$ from the 3' end of the tRNA were grown to stationary phase overnight in media lacking uracil and containing galactose. Strains were diluted to an $\mathrm{OD}_{600}$ of 0.1 and grown for 24 hours at $30^{\circ} \mathrm{C}$ with agitation in media lacking uracil and containing either galactose or glucose as indicated. $\mathrm{OD}_{600}$ was measured every 15 minutes and doubling time in minutes calculated from the growth curves. Stars indicate significant differences (Welch's T-test, $\left.{ }^{* *} \mathrm{P}<0.0005\right)$ and ns indicates the difference was not statistically significant. 


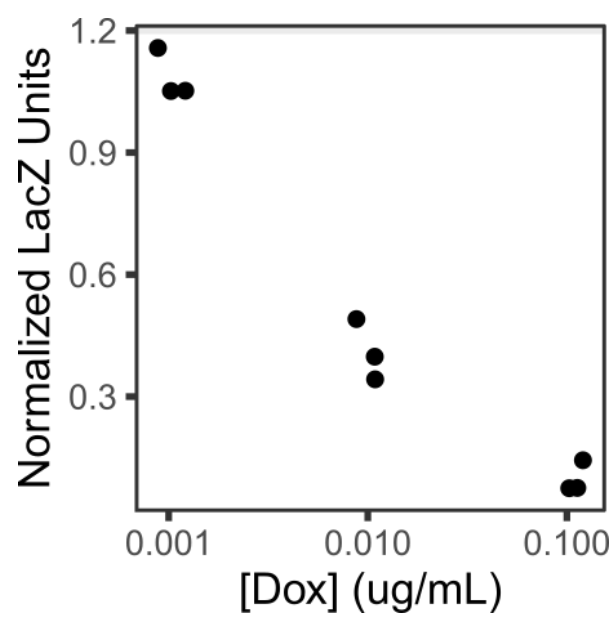

Figure S3. Effect of doxycycline concentration on transcription from the tetO promoter. CY8652 containing the TetR-VP16 activator and tetO-LacZ reporter were grown to stationary phase in medium lacking uracil and leucine. Strains were diluted in the same medium containing the indicated concentrations of doxycycline and grown for eight hours. $\beta$-galactosidase activity was determined and normalized to the strains grown in the absence of doxycycline. Each point represents one biological replicate.

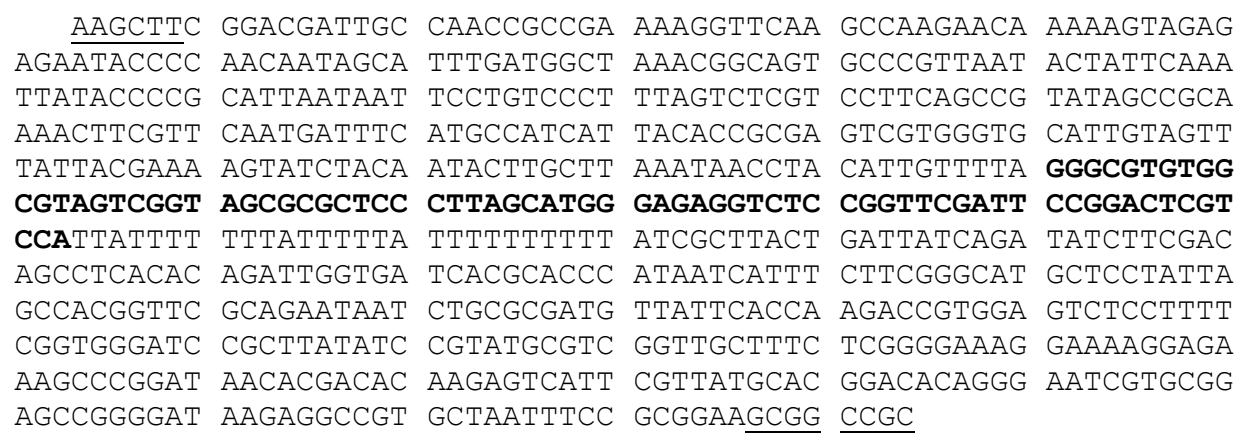

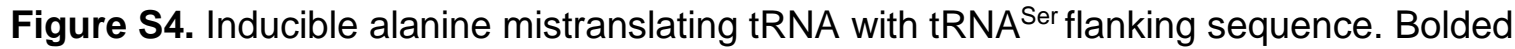
sequence represents the alanine tRNA. Underlined sequence is the restriction sites used to clone the construct into the yeast expression plasmid. 


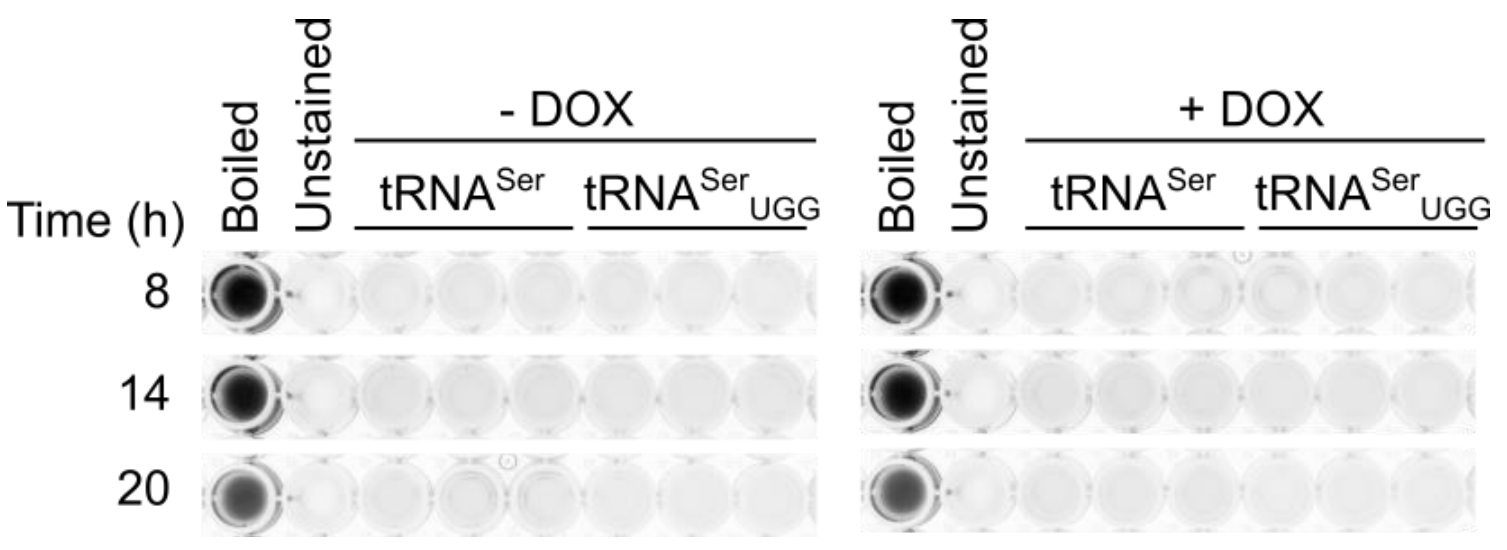

Figure S5. Inducing high levels of mistranslation does not increase propidium iodide staining.

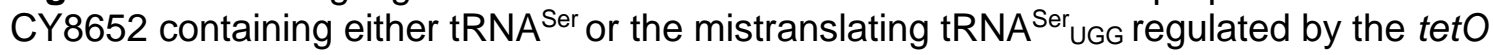
promoter were grown to stationary phase in medium lacking uracil and leucine. Strains were diluted in the same medium to an $\mathrm{OD}_{600}$ of 0.1 and grown for 6 hours before doxycycline was added to a final concentration of $10 \mu \mathrm{g} / \mathrm{mL}$. At various time points after doxycycline addition, aliquots of cells were stained with propidium iodide and imaged using a UV transilluminator. 

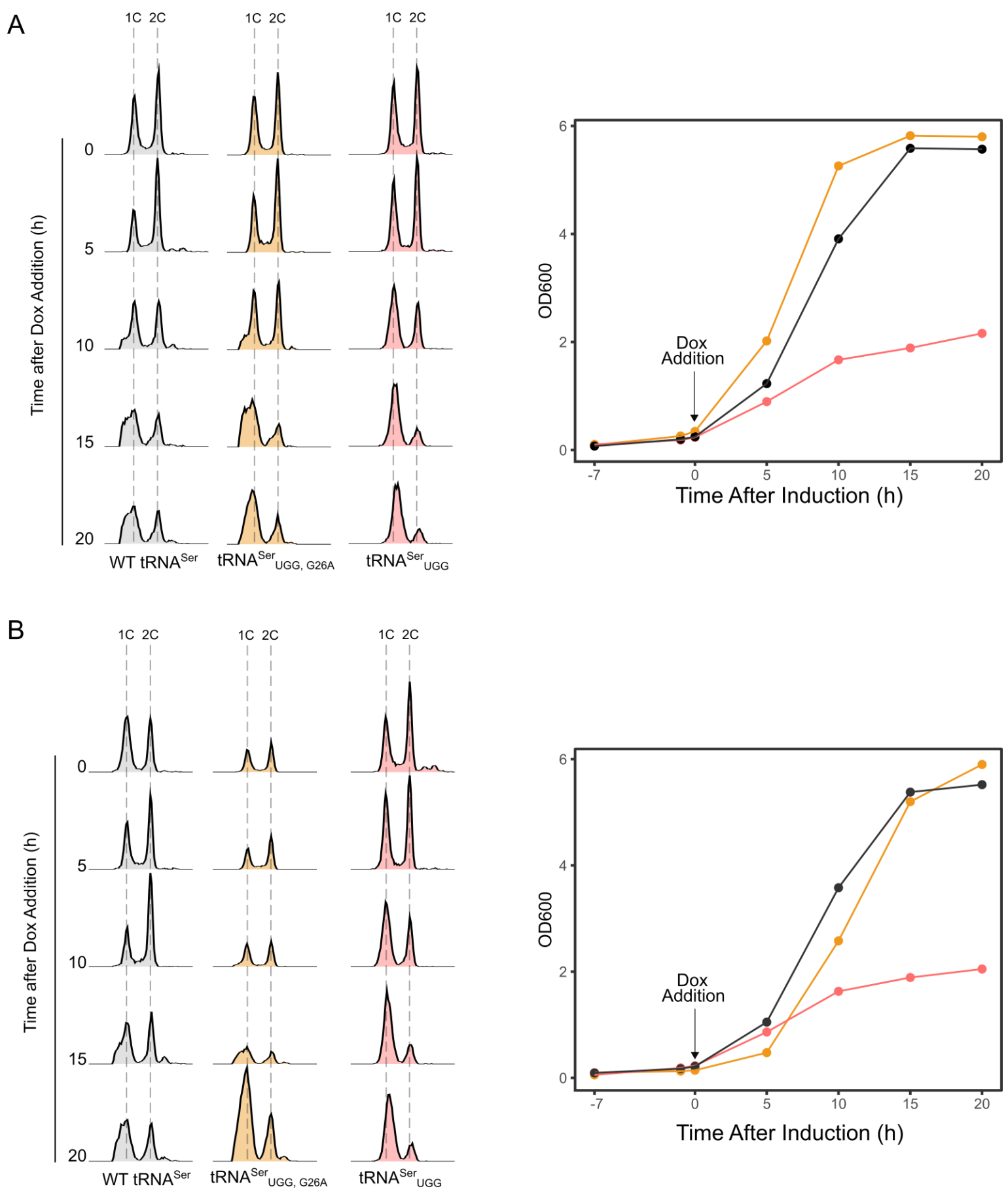

Figure S6. Flow cytometric analysis of samples taken at various time points after doxycycline addition as in Figure 6B. Two additional replicates are shown in (A) and (B) respectively. Samples were taken at the times indicated in hours following doxycycline addition, prepared for analysis and stained with propidium iodide. Histograms represent $\sim 10,000$ cells. Positions of cells with $1 \mathrm{C}$ and $2 \mathrm{C}$ DNA content are indicated on the $\mathrm{x}$ axis, which reflects fluorescence intensity on a linear scale. The $y$-axis represents cell frequency and has been scaled to represent the percentage of the maximum bin contained in that graph. On the right, growth curves of strains used for flow cytometry analysis are shown. The arrow indicates the time of doxycycline addition. 